\title{
A LAW OF THE INTEGRAED LOGARITHM FOR THE TAIL SUMS OF DYADIC MARTINGALES USING STOPPING TIMES
}

\author{
Ananta Acharya ${ }^{1}$, Santosh Ghimire ${ }^{2}$ \\ ${ }^{1}$ Central Department of Mathematics, Tribhuvan University, Kirtipur, Nepal \\ Email Address: anantaz@gmail.com \\ ${ }^{2}$ Department of Science and Humanities, Institute of Engineering, Central Campus, Pulchowk, Tribhuvan \\ University, Nepal \\ Email Address: santoshghimire@ioe.edu.np
}

\begin{abstract}
Stopping times have been used in number of places in the derivation of law of iterated logarithm for various context. In this article, we obtain a law of the iterated logarithm for the tail sums of dyadic martingales using stopping times.
\end{abstract}

Keywords: Dyadic Martingales, Tail LIL, Stopping times.

\section{Introduction}

In probability theory, the law of iterated logarithm (LIL) describes the magnitude of the fluctuation of a random walk. Its study is directly or indirectly related to dyadic interval and dyadic martingales. A dyadic interval of the unit cube $[0,1)$ is of the form $Q_{n j}=\left[\frac{j}{2^{n}}, \frac{j+1}{2^{n}}\right)$ for $n, j \in \mathbb{Z}$. Generally, we write $\mathrm{Q}_{\mathrm{n}}$ to denote a generic interval of length $\frac{1}{2^{\mathrm{n}}}$ [3]. If $\mathrm{F}_{\mathrm{n}}$ denotes the $\sigma$-algebra generated by the dyadic intervals of the form $\left[\frac{j}{2^{n}}, \frac{j+1}{2^{n}}\right)$ on $[0,1)$ then the conditional expectation of $f_{n+1}$ on $F_{n}$ is given by $E\left(f_{n+1} \mid F_{n}\right)=\frac{1}{\left|Q_{n}\right|} \int_{Q_{n}} f_{n+1}(y) d y, x \in Q_{n}$. In this consideration, a dyadic martingale is a sequence of integrable functions $\left\{f_{n}\right\}_{n=0}^{\infty}$ with $f_{n}:[0,1) \rightarrow \mathbb{R}$ such that for every $n, f_{n}$ is $F_{n}$ - measurable and $E\left(f_{n+1} \mid F_{n}\right)=f_{n}$ for all $n \geq 0$. [2]

For a dyadic martingale, we define the maximal functions as $f_{m}^{*}=\sup _{1 \leq \mathrm{k} \leq \mathrm{m}}\left|\mathrm{f}_{\mathrm{k}}\right|$ and $\mathrm{f}^{*}=$ $\sup _{1 \leq \mathrm{k}<\infty}\left|\mathrm{f}_{\mathrm{k}}\right|$ and the martingale tail square function is given as $\mathrm{S}_{\mathrm{n}}^{\prime 2} \mathrm{f}(\mathrm{x})=\left(\mathrm{S}_{\mathrm{n}}^{\prime} \mathrm{f}(\mathrm{x})\right)^{2}=$ $\sum_{k=n+1}^{\infty} d_{k}^{2}(x)$, where $d_{k}=f_{k}(x)-f_{(k-1)}(x)$ is the general term of martingale difference sequence $\left\{\mathrm{d}_{\mathrm{k}}\right\}_{1}^{\infty} \cdot[2]$

In addition, for a dyadic martingale, we have $\left\{\mathrm{x} \mathrm{f}^{*}(\mathrm{x})<\infty\right\}=\left\{\mathrm{x}: \lim \mathrm{f}_{\mathrm{n}}(\mathrm{x})\right.$ exists $\}$ a.s. [1]

In this context, a theorem on the tail LIL for dyadic martingales gives an important result which is stated in the following theorem.[4] 


\section{Theorem 1 (Tail LIL for Dyadic Martingale)}

Let $\left\{\mathrm{f}_{\mathrm{n}}\right\}_{\mathrm{n}=0}^{\infty}$ be a dyadic martingale. Assume that there exists a constant $\mathrm{C}<\infty$ such that $\left|\frac{\mathrm{S}_{\mathrm{n}}^{\prime} \mathrm{f}(\mathrm{x})}{\mathrm{s}_{\mathrm{n}}^{\prime} \mathrm{f}(\mathrm{y})}\right| \leq$ $C, \forall x, y \in I_{n j}$ for $n=1,2,3 \quad \ldots, \quad j \in\left\{\begin{array}{lllllll}0, & 1, & 2, & 3, & \ldots & 2^{n}-1\end{array}\right\}$ where $I_{n j}=\left[\frac{j}{2^{n}}, \frac{j+1}{2^{n}}\right)$. Then $\limsup _{n \rightarrow \infty} \frac{\left|f_{n}(x)-f(x)\right|}{\sqrt{2 S_{n}^{\prime 2} f(x) \log \log _{\frac{1}{S_{n}^{\prime 2} f(x)}}}} \leq 2 C$ for a. e. $x$.

From the assumption, we get $\operatorname{Sf}(x)<\infty$ for a.e. $x$. This shows that the sequence $\left\{\mathrm{f}_{\mathrm{n}}(\mathrm{x})\right\}$ converges [1]. Thus the tail law of the iterated logarithm gives the rate of convergence of dyadic martingales $\left\{f_{n}\right\}$ to its limit function $\mathrm{f}$. Moreover, the rate of convergence depends on the tail sums of martingale square function.

As continuation in the tail LIL for dyadic martingales, we obtained a new result which can be considered as the corollary of the theorem on tail LIL for dyadic martingales stated above. Our main result is as follows.

Theorem $2 \operatorname{Let}\left\{\mathrm{f}_{\mathrm{n}}\right\}_{\mathrm{n}=0}^{\infty}$ be a dyadic martingale. Fix $\theta>1$. Define stopping times $\mathrm{n}_{\mathrm{k}}(\mathrm{x})=$ $\min \left\{n: x \in I_{n_{j}}\right.$, for some $j \in\left\{1,2,3, \ldots, 2^{n}\right\}$ and $\left.\forall y \in I_{n_{j}}, S_{n}^{\prime} f(y)<\frac{1}{\theta^{k}}\right\}$. Then for the sequence of stopping times $\mathrm{n}_{\mathrm{k}}(\mathrm{x})$,

$$
\limsup _{\mathrm{k} \rightarrow \infty} \frac{\left|\mathrm{f}(\mathrm{x})-\mathrm{f}_{\mathrm{n}_{\mathrm{k}}}(\mathrm{x})\right|}{\sqrt{2 \mathrm{~S}_{\mathrm{n}}^{\prime 2} \mathrm{f}(\mathrm{x}) \log \log \frac{1}{{S_{\mathrm{n}}^{\prime}}^{2} \mathrm{f}(\mathrm{x})}}}<\sqrt{3}
$$

for a.e. $\mathrm{x}$.

Proof:

First of all we prove the following estimate for $\lambda>0, \eta>0$,

$$
\left|\left\{x \in[0,1):\left|f(x)-f_{n}(x)\right|>\lambda, S_{n}^{\prime} f(x)<\eta \lambda\right\}\right| \leq \exp \left(\frac{-1}{2 \eta^{2}}\right)
$$

To prove this we have

$$
\left|\left\{x:\left|f(x)-f_{n}(x)\right|>\lambda\right\}\right| \leq 6 \exp \left(\frac{-\lambda^{2}}{2|| S_{n}^{\prime} f||_{\infty}^{2}}\right)
$$

Here, $S_{n}^{\prime} f(x)<\eta \lambda$ gives $\left\|S_{n}^{\prime} f\right\|_{\infty}^{2} \leq \eta^{2} \lambda^{2}$. So, $\frac{-1}{\left\|S_{n}^{\prime} f\right\|_{\infty}^{2}} \leq \frac{-1}{\eta^{2} \lambda^{2}}$. So we have,

$$
\begin{aligned}
\left|\left\{x \in[0,1):\left|f(x)-f_{n}(x)\right|>\lambda, S_{n}^{\prime} f(x)<\eta \lambda\right\}\right| & \leq 6 \exp \left(\frac{-\lambda^{2}}{2|| S_{n}^{\prime} f||_{\infty}^{2}}\right) \\
& \leq 6 \exp \left(\frac{-\lambda^{2}}{2 \eta^{2} \lambda^{2}}\right) \\
& =\exp \left(\frac{-1}{2 \eta^{2}}\right)
\end{aligned}
$$


This is the required result (1).

Now, choose $\lambda=\frac{(1+\epsilon) \sqrt{2 \log \log \theta^{21}}}{\theta^{2}}$ and $\eta=\frac{\theta}{(1+\epsilon) \sqrt{2 \log \log \theta^{21}}}$ where $\theta>1$ and $\epsilon>0$. Then using (1) we have,

$$
\begin{aligned}
\mid\left\{x \in[0,1):\left|f(x)-f_{n}(x)\right|>\frac{(1+\epsilon) \sqrt{2 \log \log \theta^{21}}}{\theta^{2}}\right. & \left., S_{n}^{\prime} f(x)<\frac{1}{\theta^{1-1}}\right\} \mid \\
& \leq 6 \exp \left(\frac{-(1+\epsilon)^{2}\left(2 \log \log \theta^{21}\right.}{2 \theta^{2}}\right) \\
& =6 \exp \left(\log (2 \log \theta)^{\frac{-(1+\epsilon)^{2}}{\theta^{2}}}\right) \\
& =6(2 \log \theta)^{\frac{-(1+\epsilon)^{2}}{\theta^{2}}} \\
& =\frac{6}{(2 \log \theta)^{\frac{(1+\epsilon)^{2}}{\theta^{2}}}} \\
& =\frac{6}{(2 \log \theta)^{\frac{(1+\epsilon)^{2}}{\theta^{2}}} \cdot\left(\frac{1}{1}\right)^{\frac{(1+\epsilon)^{2}}{\theta^{2}}}}
\end{aligned}
$$

Let us choose $\epsilon=\sqrt{3} \theta-1$. Then we have $\frac{(1+\epsilon)^{2}}{\theta^{2}}=3$. Thus,

$$
\begin{gathered}
\left|\left\{x \in[0,1):\left|f(x)-f_{n}(x)\right|>\frac{(1+\epsilon) \sqrt{2 \log \log \theta^{2 l}}}{\theta^{2}}, S_{n}^{\prime} f(x)<\frac{1}{\theta^{1-1}}\right\}\right| \leq 6\left(\frac{1}{2 \log \theta}\right)^{3} \cdot \frac{1}{1^{3}} \\
=\frac{C}{1^{3}} \text { (suppose). }
\end{gathered}
$$

Now, let $(\mathrm{x})=\sqrt{\mathrm{x} \log \log \frac{1}{\mathrm{x}}}$. Then $\mathrm{g}(\mathrm{x})$ is an increasing function. So for $\frac{1}{\theta^{21}} \leq \mathrm{S}_{\mathrm{n}}^{\prime 2} \mathrm{f}(\mathrm{x})$, we have,

$$
\sqrt{2 S_{n}^{\prime 2} f(x) \log \log \frac{1}{{S_{n}^{\prime}}^{2} f(x)}} \geq \sqrt{2 \frac{1}{\theta^{2 l}} \log \log \theta^{2 l}}
$$

Now, using (3), we have,

$$
\begin{aligned}
& \left|\left\{\mathrm{x} \in[0,1):\left|\mathrm{f}(\mathrm{x})-\mathrm{f}_{\mathrm{n}}(\mathrm{x})\right|>(1+\epsilon) \sqrt{2 \mathrm{~S}_{\mathrm{n}}^{\prime 2} \mathrm{f}(\mathrm{x}) \log \log \frac{1}{\mathrm{~S}_{\mathrm{n}}^{\prime 2} \mathrm{f}(\mathrm{x})}}\right\}\right| \\
& =\left|\mathrm{U}_{\mathrm{l}=\mathrm{k}+1}^{\infty}\left\{\mathrm{x} \in[0,1):\left|\mathrm{f}(\mathrm{x})-\mathrm{f}_{\mathrm{n}}(\mathrm{x})\right|>(1+\epsilon) \sqrt{2 \mathrm{~S}_{\mathrm{n}}^{\prime 2} \mathrm{f}(\mathrm{x}) \log \log \frac{1}{\mathrm{~S}_{\mathrm{n}}^{2} \mathrm{f}(\mathrm{x})}}, \frac{1}{\theta^{1}} \leq \mathrm{S}_{\mathrm{n}}^{\prime} \mathrm{f}(\mathrm{x})<\frac{1}{\theta^{1-1}}\right\}\right| \\
& \leq\left|\mathrm{U}_{\mathrm{l}=\mathrm{k}+1}^{\infty}\left\{\mathrm{x} \in[0,1):\left|\mathrm{f}(\mathrm{x})-\mathrm{f}_{\mathrm{n}}(\mathrm{x})\right|>(1+\epsilon) \sqrt{2 \frac{1}{\theta^{21}} \log \log \theta^{21}}, \mathrm{~S}_{\mathrm{n}}^{\prime} \mathrm{f}(\mathrm{x})<\frac{1}{\theta^{1-1}}\right\}\right| \\
& =\left|\mathrm{U}_{\mathrm{l}=\mathrm{k}+1}^{\infty}\left\{\mathrm{x} \in[0,1):\left|\mathrm{f}(\mathrm{x})-\mathrm{f}_{\mathrm{n}}(\mathrm{x})\right|>\frac{1+\epsilon}{\theta^{1}} \sqrt{2 \log \log \theta^{21}}, \mathrm{~S}_{\mathrm{n}}^{\prime} \mathrm{f}(\mathrm{x})<\frac{1}{\theta^{1-1}}\right\}\right| \\
& \leq \sum_{\mathrm{l}=\mathrm{k}+1}^{\infty}\left|\left\{\mathrm{x} \in[0,1):\left|\mathrm{f}(\mathrm{x})-\mathrm{f}_{\mathrm{n}}(\mathrm{x})\right|>\frac{1+\epsilon}{\theta^{1}} \sqrt{2 \log \log \theta^{21}}, \mathrm{~S}_{\mathrm{n}}^{\prime} \mathrm{f}(\mathrm{x})<\frac{1}{\theta^{1-1}}\right\}\right|
\end{aligned}
$$


$\leq \sum_{\mathrm{l}=\mathrm{k}+1}^{\infty} \frac{\mathrm{C}}{\mathrm{l}^{3}}$

We know that,

$$
\sum_{\mathrm{l}=\mathrm{k}+1}^{\infty} \frac{1}{\mathrm{l}^{3}} \leq \int_{\mathrm{k}}^{\infty} \frac{1}{\mathrm{x}^{3}} \mathrm{dx}=\left[\frac{-1}{2 \mathrm{x}^{2}}\right]_{\mathrm{k}}^{\infty}=\frac{1}{\mathrm{k}^{2}}
$$

So, (4) can be written as,

$$
\left|\left\{x \in[0,1):\left|f(x)-f_{n}(x)\right|>(1+\epsilon) \sqrt{2 S_{n}^{\prime 2} f(x) \log \log \frac{1}{S_{n}^{\prime 2} f(x)}}\right\}\right| \leq \frac{C}{k^{2}}
$$

This can be done for every $\mathrm{n}_{\mathrm{k}}(\mathrm{x})$. So summing over all $\mathrm{k}$ we have,

$$
\begin{aligned}
\sum_{\mathrm{k}=1}^{\infty}\left|\left\{\mathrm{x} \in[0,1):\left|\mathrm{f}(\mathrm{x})-\mathrm{f}_{\mathrm{n}}(\mathrm{x})\right|>(1+\epsilon) \sqrt{2 \mathrm{~S}_{\mathrm{n}}^{\prime 2} \mathrm{f}(\mathrm{x}) \log \log \frac{1}{\mathrm{~S}_{\mathrm{n}}^{\prime 2} \mathrm{f}(\mathrm{x})}}\right\}\right| & \leq \sum_{\mathrm{k}=1}^{\infty} \frac{\mathrm{C}}{\mathrm{k}^{2}} \\
& =\mathrm{C} \sum_{\mathrm{k}=1}^{\infty} \frac{1}{\mathrm{k}^{2}}<\infty
\end{aligned}
$$

So, by Borel Cantelli lemma, for a.e. $\mathrm{x}$, there exists $\mathrm{M}$ which depends on $\mathrm{x}$ such that for every $\mathrm{k} \geq \mathrm{M}$,

$$
\left|f(x)-f_{n_{k}}(x)\right| \leq(1+\epsilon) \sqrt{2 S_{n}^{\prime 2} f(x) \log \log \frac{1}{S_{n}^{\prime 2} f(x)}}
$$

But we have choosen $\epsilon=\sqrt{3} \theta-1$. So,

$$
\left|f(x)-f_{n_{k}}(x)\right| \leq \sqrt{3} \theta \sqrt{2 S_{n}^{\prime 2} f(x) \log \log \frac{1}{S_{n}^{\prime 2} f(x)}}
$$

that is,

$$
\frac{\left|f(x)-f_{n_{k}}(x)\right|}{\sqrt{2 S_{n}^{\prime 2} f(x) \log \log \frac{1}{S_{n}^{\prime 2} f(x)}}} \leq \sqrt{3} \theta
$$

It is noted that as $\mathrm{n} \rightarrow \infty, \mathrm{k} \rightarrow \infty$. Now, letting $\theta \downarrow 1$, we get for a. e. $\mathrm{x}$,

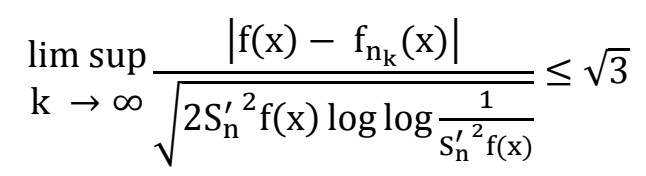




\section{References}

1. Burkholder D.L. and Gundy R.F., "Extrapolation and interpolation of quasi-linear operators on martingales", Acta Mathematics 124, 249- 304, (1970)

2. Ghimire S., "The Law of Iterated Logarithm for Tail sums", Ph.D. Dissertation, Kansas University, USA, (2012)

3. Ghimire S. and Moore C.N., "A lower bound in the tail law of the iterated logarithm for lacunary trigonometric series", Proceedings of the American Mathematical Society.142 (9): 3207-3216, (2014)

4. Stout W., "A martingale Analogue of Kolmogorov's law of the iterated logarithm”, Z. Wahrscheinlichkeitstheorie verw. Geb. 15: 279- 290, (1970) 